\title{
The Reading the Mind in the Eyes Test: Psychometric properties in a Peruvian Population
}

Zegarra- Valdivia, J. \& Chino, B.

Universidad Nacional de San Agustín de Arequipa. Perú

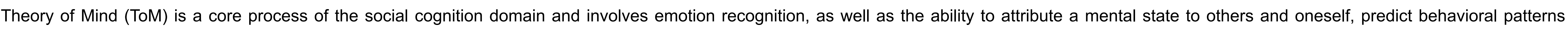

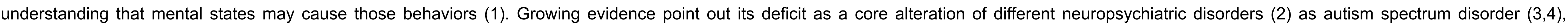

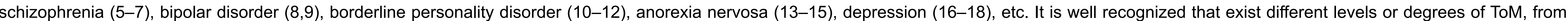

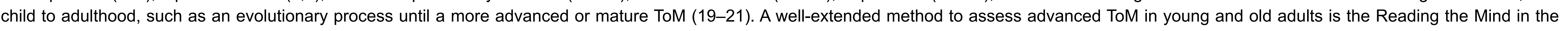

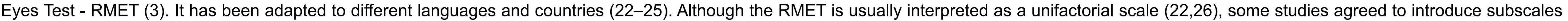
related to the emotion expression.

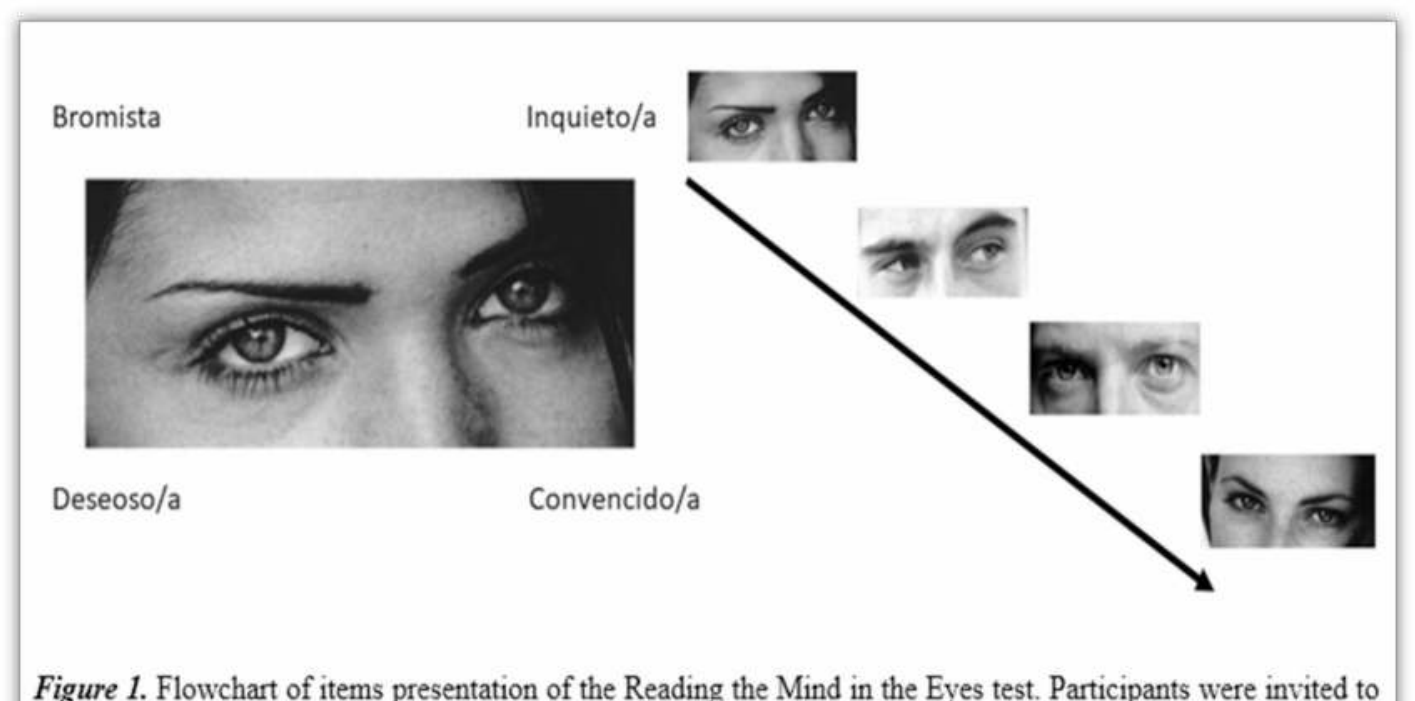
Figure 1. Flowchart of items presentation of the Reading the Mind in the Eyes test. Participants were invited to
answer which is the gender of the person in each picture, and also what is he or she feeling or thinking (choosing
one of the four possibile options around).

Table 2

Correct answer distribution for different versions of the Reading the Mind in the Eyes Test
from European and Non-European countries.

\begin{tabular}{|c|c|c|c|c|c|c|c|c|}
\hline Item & Peruvia & $\begin{array}{c}\text { Brazilian } \\
(200)\end{array}$ & Spanish & Italia & German & $\begin{array}{c}\text { French } \\
(27)\end{array}$ & English & Persian \\
\hline Q1 & $\begin{array}{c}n \\
42.6\end{array}$ & $\begin{array}{l}(39) \\
37.1\end{array}$ & $\begin{array}{l}(38) \\
66.9\end{array}$ & & & & & \\
\hline & 84.4 & 42.9 & 63.8 & 56 & 49.4 & 70 & 78.7 & $\begin{array}{l}72.61 \\
72\end{array}$ \\
\hline Q3 & 77.2 & 77.7 & 75.2 & 65 & 85.1 & 93 & 86.1 & 53.4 \\
\hline Q4 & 63.5 & 66.4 & 81.1 & 65.5 & 74.2 & 54 & 73 & 63.7 \\
\hline Q5 & $\begin{array}{l}70.3 \\
67.3\end{array}$ & $\begin{array}{c}75 \\
786\end{array}$ & $\begin{array}{l}92.5 \\
72.2\end{array}$ & 84 & 64.5 & 71 & 77 & 58.2 \\
\hline $\begin{array}{l}\text { Q6 } \\
07\end{array}$ & $\begin{array}{l}6.3 \\
62.7\end{array}$ & $\begin{array}{l}78.6 \\
51.8\end{array}$ & $\begin{array}{l}75.2 \\
64.6\end{array}$ & $\begin{array}{c}69 \\
42.5\end{array}$ & $\begin{array}{l}72.9 \\
49\end{array}$ & $\begin{array}{l}80 \\
33\end{array}$ & $\begin{array}{l}80.3 \\
68\end{array}$ & $\begin{array}{l}78.3 \\
18.7\end{array}$ \\
\hline & $\begin{array}{l}0.2 .1 \\
63.5 \\
6.5\end{array}$ & 80.4 & $\begin{array}{c}64.6 \\
88\end{array}$ & $\begin{array}{c}42.5 \\
67\end{array}$ & $\begin{array}{c}49 \\
77.4\end{array}$ & $\begin{array}{l}33 \\
68\end{array}$ & $\begin{array}{c}68 \\
67.2\end{array}$ & $\begin{array}{l}18.7 \\
72.7\end{array}$ \\
\hline 09 & 56.3 & $\begin{array}{l}80.4 \\
56.5\end{array}$ & $\begin{array}{l}88 \\
81.9\end{array}$ & 90.5 & $\begin{array}{l}71.4 \\
78.6\end{array}$ & $\begin{array}{l}80 \\
81\end{array}$ & ${ }_{77}^{0.2}$ & $\begin{array}{l}61.1 \\
61.1\end{array}$ \\
\hline Q10 & 54.8 & 51 & 71 & 63.5 & 76 & 60 & 73 & 43.9 \\
\hline Q11 & 62.7 & 77 & 74.1 & 71 & 74.3 & 57 & 68 & 52.1 \\
\hline $\begin{array}{l}\text { Q12 } \\
\mathrm{O13}^{2}\end{array}$ & $\begin{array}{r}51 \\
639\end{array}$ & $\begin{array}{l}44.1 \\
{ }_{7}^{2}\end{array}$ & 80 & $\begin{array}{l}71.5 \\
7.1\end{array}$ & $\begin{array}{l}87.7 \\
8\end{array}$ & 75 & 87.7 & 74.3 \\
\hline & $\begin{array}{l}63.9 \\
56.7\end{array}$ & $\begin{array}{l}777 \\
75\end{array}$ & $\begin{array}{l}80.8 \\
88.9\end{array}$ & $\begin{array}{l}63.5 \\
80\end{array}$ & $\begin{array}{l}55.8 \\
734\end{array}$ & $\begin{array}{l}34 \\
{ }_{85}\end{array}$ & $\begin{aligned} 69.7 \\
8\end{aligned}$ & $\begin{array}{l}64 \\
936\end{array}$ \\
\hline Q15 & & 91.9 & 86.9 & 83 & 84.5 & & $\begin{array}{l}60.5 \\
69.7\end{array}$ & 80.7 \\
\hline 0 & 56.3 & 60.3 & 85.8 & 76 & 76 & 79 & 77 & 5 \\
\hline Q17 & 63.1 & 48.8 & 54.3 & 54 & 50.3 & 48 & 65.6 & 57.8 \\
\hline Q18 & 81.7 & 87.2 & 96.4 & 92 & 81.9 & 86 & 58.2 & 82.4 \\
\hline $\begin{array}{l}\text { Q19 } \\
\text { O9. }\end{array}$ & $\begin{array}{l}19.4 \\
738 .\end{array}$ & 59.2 & $\begin{array}{c}39 \\
894\end{array}$ & 525 & $\begin{array}{l}57.4 \\
\text { S. }\end{array}$ & $\begin{array}{l}43 \\
{ }_{23}\end{array}$ & $\begin{array}{l}69.7 \\
\text { 96. }\end{array}$ & 69.7 \\
\hline $\begin{array}{l}\mathrm{Q} 20 \\
\mathbf{0 2 1}\end{array}$ & $\begin{array}{l}73.8 \\
75.7\end{array}$ & $\begin{array}{c}77 \\
77.3\end{array}$ & $\begin{array}{l}89.4 \\
75.2\end{array}$ & $\begin{array}{c}73.5 \\
73\end{array}$ & $\begin{array}{l}81.3 \\
39.4\end{array}$ & $\begin{array}{l}92 \\
86\end{array}$ & $\begin{array}{l}88.5 \\
7\end{array}$ & $\begin{array}{l}91.4 \\
561\end{array}$ \\
\hline 022 & 77.2 & 88.7 & 70.8 & 90.5 & 72.9 & & & 80.7 \\
\hline Q23 & 59.3 & 64.2 & 65.5 & 62.5 & 61.7 & 37 & 77.9 & 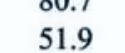 \\
\hline Q24 & 62 & 68.1 & 73.5 & 58.5 & 57.4 & 84 & 73.8 & 64.6 \\
\hline Q25 & 27.4 & 68.3 & 70.5 & 67 & 42.6 & 76 & 71.3 & 39.3 \\
\hline $\begin{array}{l}\text { Q26 } \\
\text { 27 }\end{array}$ & $\begin{array}{l}44.1 \\
{ }_{21}\end{array}$ & 62.88 & $\begin{array}{l}75.2 \\
75.2\end{array}$ & $\begin{array}{l}76.5 \\
6.5\end{array}$ & $\begin{array}{l}78.1 \\
7\end{array}$ & $\begin{array}{l}68 \\
{ }_{10}\end{array}$ & 65.6 & 78 \\
\hline $\begin{array}{l}\text { Q27 } \\
028\end{array}$ & $\begin{array}{l}37.3 \\
77.6\end{array}$ & $\begin{array}{c}43.2 \\
72\end{array}$ & $\begin{array}{l}64.1 \\
83.6\end{array}$ & $\begin{array}{l}63 \\
70\end{array}$ & $\begin{array}{l}6.1 .1 \\
6\end{array}$ & 49 & & $\begin{array}{l}47.5 \\
47\end{array}$ \\
\hline & 61.2 & 74.2 & 81.1 & 66.5 & 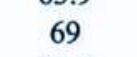 & 66 & 77.9 & \\
\hline Q30 & 84 & 90.5 & 88.6 & 91 & 86.5 & 80 & 91 & 86.8 \\
\hline & 70.3 & 62.5 & 57.1 & 66.5 & 32.3 & 69 & 51.6 & 58.3 \\
\hline Q32 & 73.4 & 56.4 & & 73 & 66.5 & 80 & 0 & 79.1 \\
\hline & $\begin{array}{l}30.4 \\
329\end{array}$ & 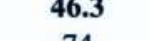 & $\begin{array}{ll}6.3 \\
7\end{array}$ & 54. & $\begin{array}{l}77.4 \\
77\end{array}$ & 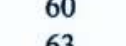 & $\begin{array}{l}58.2 \\
87\end{array}$ & 66.6 \\
\hline & 529 & 423 & 777 & 36.5 & 606 & 4 & 6 & 53 \\
\hline 036 & 62 & 89 & 87.5 & 76.5 & 85.8 & 71 & 76.2 & 68.6 \\
\hline
\end{tabular}

\section{OBJECTIVE}

The aim of this study is to analyze the psychometric properties of the Reading the mind in the eyes in the Peruvian population, as well as the test-retest reliability after one-year follow-up.
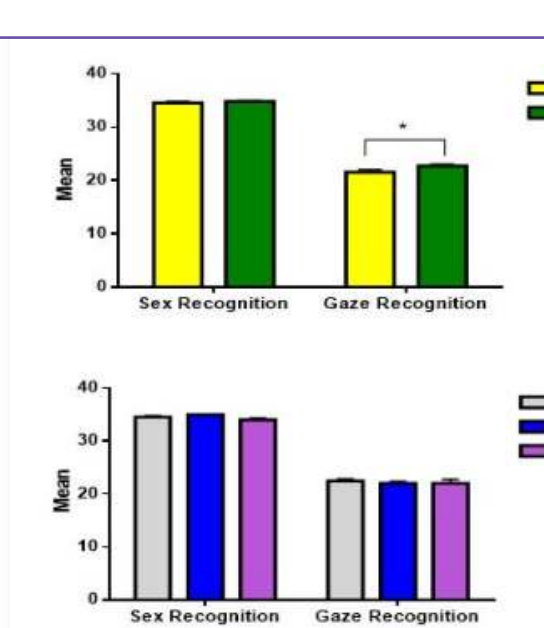

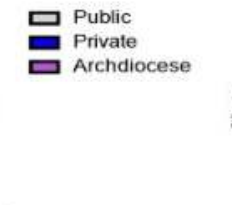
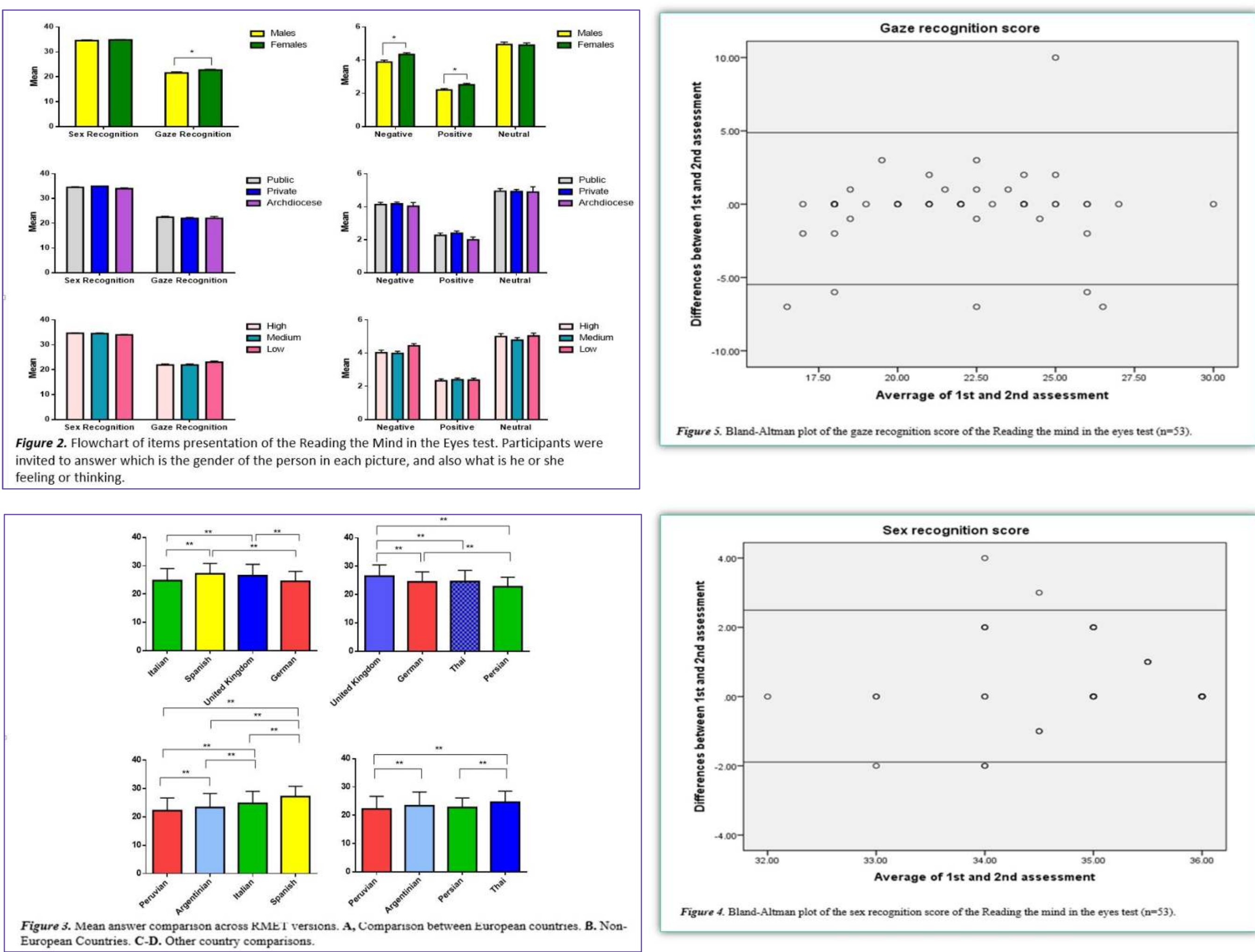

DISCUSSION

The reliability of the Reading the Mind in the Eyes Peruvian version has good. Also, test-retest validity after one-year follow-up study shows a good consistency in gaze and sex scores, similarly to the Persian and Spanish version $(24,38)$. ICC and Bland-Altman plot also shows that RMET has stable scores between tests to retest assess, even after one-year follow-up. Item-Item analysis displayed a pattern of equivalent functioning to other internationa studies.

Regarding sex differences, our study confirms one more time that females scored higher than males participants $(22,33,42)$; even in positive or negative faces. We did not find differences in neutral faces More importantly, we did not find differences in socio-economic status or education, even comparing

for negative/positive/neutral faces, despite the wide ranges of age evaluated, and the demographic differences.

These results may improve clinical practice and further studies in Peru may discriminate better between clinical and nonclinical samples.
Acknowledgment: Zegarra, J. \& Chino, B. recognize the financial support of the Consejo Nacional de Ciencia Tecnología e Innovación Tecnología (Concytec) through its executing unit Fondo Nacional de Desarrollo Científico, Tecnológico y de Innovación Tecnológica (Fondecyt) for their research work.

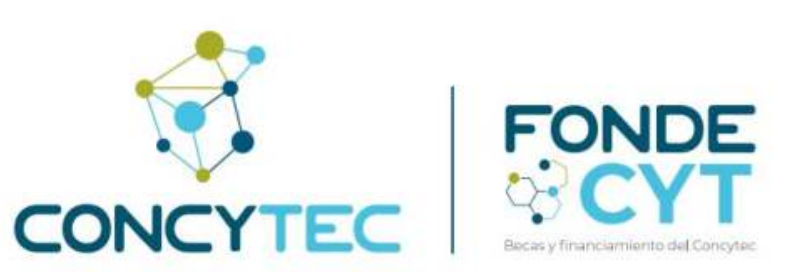

